\title{
On the Splitting Parameter in the Ewald Method
}

\author{
Alp Kustepeli and Anthony Q. Martin
}

\begin{abstract}
An investigation of the Ewald method is presented. The method involves a splitting parameter that is theoretically an arbitrary number. An analysis is presented to show why the splitting parameter cannot always be treated as arbitrary in calculations and how this parameter should be chosen for all periodic spacing of a structure.
\end{abstract}

Index Terms-Acceleration, periodic structures, planar arrays, series, waveguides.

\section{INTRODUCTION}

$\mathbf{T}$ HE efficient evaluation of Green's functions involving slowly convergent series is an important issue for the analysis of many structures by integral equation methods. In waveguide, cavity, or periodic array problems, one may use the free-space periodic Green's function (FSPGF) as the kernel of integral equations. The Ewald method [1] is a powerful means to efficiently evaluate the FSPGF. In the Ewald method the FSPGF is expressed as the sum of a "spectral" and a "spatial" series. The terms of these series possess Gaussian decay and this leads to a series representation that exhibits a very rapid convergence rate.

In the evaluation of the FSPGF for two-dimensional (2-D) periodic arrays with the Ewald method, the spectral and the spatial series, which are written in terms of the complementary error function, involve a splitting parameter $E$. In [2] it is stated that $E$ is an arbitrary number and its optimum value, $E_{\text {opt }}$, is given to balance the convergence rate between these two series thereby causing the total number of terms needed for the calculation to be minimized. If $E$ is increased beyond $E_{o p t}$ then successive terms in the spatial series decay faster while successive terms in the spectral series decay slower. In studies involving the Ewald method [2], [3], the $E_{\text {opt }}$ value is generally used to sum the spectral and the spatial series arising in the analysis of structures having small periodic spacing. Although the splitting parameter is theoretically arbitrary, we show that one cannot get correct results even when $E=E_{\text {opt }}$ for structures having large enough periodic spacing. Therefore the splitting parameter should always be chosen carefully in calculations. One can also apply the Ewald method for the calculation of the FSPGF due to three-dimensional (3-D) periodic arrays [1]. In [4], for 3-D, a value of $E$ is first determined by examining only the convergence of

Manuscript received June 7, 1999; revised March 20, 2000. This work was supported in part by the Air Force Office of Scientific Research, Bolling AFB, Wasshington, DC, Grant 49620-96-1-0005

A. Kustepeli was with the Holcombe Department of Electrical and Computer Engineering, Clemson University, Clemson, SC 29634 USA. He is now with the Electrical and Electronics Engineering Department, Izmir Institute of Technology, Izmir, Turkey.

A. Q. Martin is with the Holcombe Department of Electrical and Computer Engineering, Clemson University, Clemson, SC 29631 USA.

Publisher Item Identifier S 1051-8207(00)06377-7. the spatial series and this value is used in the computations. As stated for 2-D arrays, the choice of this parameter is also very important in 3-D for balancing the convergence rate in the analysis of structures with small periodic spacing and for obtaining correct values in the analysis of structures having large periodic spacing.

In this letter we present an expression for $E_{\text {opt }}$ which can be used for the calculation of the FSPGF for 3-D periodic arrays of small periodic spacing. We compare results obtained by the Ewald method with those obtained by the well-known Shanks' transform [5] computed by Wynn's $\varepsilon$-algorithm [6]. We demonstrate the importance of the splitting parameter, comment on how it should be chosen for large spacing, and show the efficiency of the Ewald method with carefully chosen $E$.

\section{EWALD METHOD}

The FSPGF for 2-D periodic arrays is given by

$$
G\left(r, r^{\prime}\right)=\frac{1}{4 \pi} \sum_{m, n=-\infty}^{\infty} \frac{e^{-j k R_{m n}}}{R_{m n}}
$$

where

$$
R_{m n}=\sqrt{\left(\xi-m D_{x}\right)^{2}+\left(\eta-n D_{y}\right)^{2}+\zeta^{2}}
$$

with

$$
\xi=x-x^{\prime}, \quad \eta=y-y^{\prime}, \quad \zeta=z-z^{\prime} .
$$

The term $R_{m n}$ in (1) represents the distance between the observation point at $(x, y, z)$ and the periodic source points located in the $z^{\prime}$ plane. The quantities $D_{x}$ and $D_{y}$ represent the periodic spacing of the structure in the $x$ and $y$ directions, respectively. For 3-D the FSPGF is given by

$$
G\left(r, r^{\prime}\right)=\frac{1}{4 \pi} \sum_{m, n, p=-\infty}^{\infty} \frac{e^{-j k R_{m n p}}}{R_{m n p}}
$$

where

$$
R_{m n p}=\sqrt{\left(\xi-m D_{x}\right)^{2}+\left(\eta-n D_{y}\right)^{2}+\left(\zeta-p D_{z}\right)^{2}}
$$

When employing the Ewald method for the evaluation of (1), the Green's function is expressed as a sum of two series such that

$$
G=G_{1}+G_{2}
$$

In (6), $G_{1}$ and $G_{2}$ utilize the complementary error function, $\operatorname{erfc}(z)$. By virtue of the presence of the complementary error function in these series, a very rapid convergence rate, resulting from the Gaussian decay found in the terms of the series, is achieved. Since Gaussian decay is involved in each series, the 
value of the summation is generally obtained in a very small number of terms if $E$ is properly chosen. The spectral series $G_{1}$ is given by

$$
\begin{aligned}
G_{1}= & \frac{1}{8 D_{x} D_{y}} \sum_{m, n=-\infty}^{\infty} \frac{e^{-j 2 \pi\left(\left(m \xi / D_{x}\right)+\left(n \eta / D_{y}\right)\right)}}{\alpha_{m n}} \\
& \cdot\left[e^{2 \alpha_{m n} \zeta} \operatorname{erfc}\left(\frac{\alpha_{m n}}{E}+\zeta E\right)\right. \\
& \left.+e^{-2 \alpha_{m n} \zeta} \operatorname{erfc}\left(\frac{\alpha_{m n}}{E}-\zeta E\right)\right]
\end{aligned}
$$

where

$$
\alpha_{m n}=\left\{\begin{array}{c}
\sqrt{\left(\frac{m \pi}{D_{x}}\right)^{2}+\left(\frac{n \pi}{D_{y}}\right)^{2}-\frac{k^{2}}{4}}, \\
\left(\frac{m \pi}{D_{x}}\right)^{2}+\left(\frac{n \pi}{D_{y}}\right)^{2}>\frac{k^{2}}{4} \\
j \sqrt{\frac{k^{2}}{4}-\left[\left(\frac{m \pi}{D_{x}}\right)^{2}+\left(\frac{n \pi}{D_{y}}\right)^{2}\right]}, \\
\left(\frac{m \pi}{D_{x}}\right)^{2}+\left(\frac{n \pi}{D_{y}}\right)^{2}<\frac{k^{2}}{4}
\end{array} .\right.
$$

The spatial series $G_{2}$ is given by

$$
\begin{aligned}
G_{2}= & \frac{1}{4 \pi} \sum_{m, n=-\infty}^{\infty} \frac{1}{R_{m n}} \\
& \cdot \operatorname{Re}\left(e^{-j k R_{m n}} \operatorname{erfc}\left(R_{m n} E+\frac{-j k}{2 E}\right)\right) .
\end{aligned}
$$

For 3-D the Green's function in (4) is also expressed as a sum of two series as given in (6). In this case the spectral series $G_{1}$ is written as

$$
\begin{aligned}
G_{1}= & \frac{1}{4 D_{x} D_{y} D_{z}} \sum_{m, n, p=-\infty}^{\infty} \\
& \cdot e^{-j 2 \pi\left(\left(m \xi / D_{x}\right)+\left(n \eta / D_{y}\right)+\left(n \zeta / D_{z}\right)\right)} \frac{e^{-\alpha_{m n p}^{2} / E^{2}}}{\alpha_{m n p}^{2}}
\end{aligned}
$$

where

$$
\alpha_{m n p}^{2}=\left(m \pi / D_{x}\right)^{2}+\left(n \pi / D_{y}\right)^{2}+\left(p \pi / D_{z}\right)^{2}-k^{2} / 4
$$

and the spatial series $G_{2}$ is written as

$$
\begin{aligned}
G_{2}= & \frac{1}{4 \pi} \sum_{m, n, p=-\infty}^{\infty} \frac{1}{R_{m n p}} \\
& \cdot \operatorname{Re}\left(e^{-j k R_{m n n} \operatorname{erfc}}\left(R_{m n p} E+\frac{-j k}{2 E}\right)\right) .
\end{aligned}
$$

The optimum splitting parameter $E_{\text {opt }}$, used for the computation of (7) and (9), is given in [2] as

$$
E_{\text {opt }}=\sqrt{\frac{\pi}{D_{x} D_{y}}}
$$

TABLE I

$\log _{10}$ OF RELATIVE DIFFERENCE

BETWEen the RESUlTS OBTAINED By THE EWALD METHOD AND THE SHANKS' TRANSFORM FOR $G$ OF (1) FOR VARIOUS VALUES OF $E$

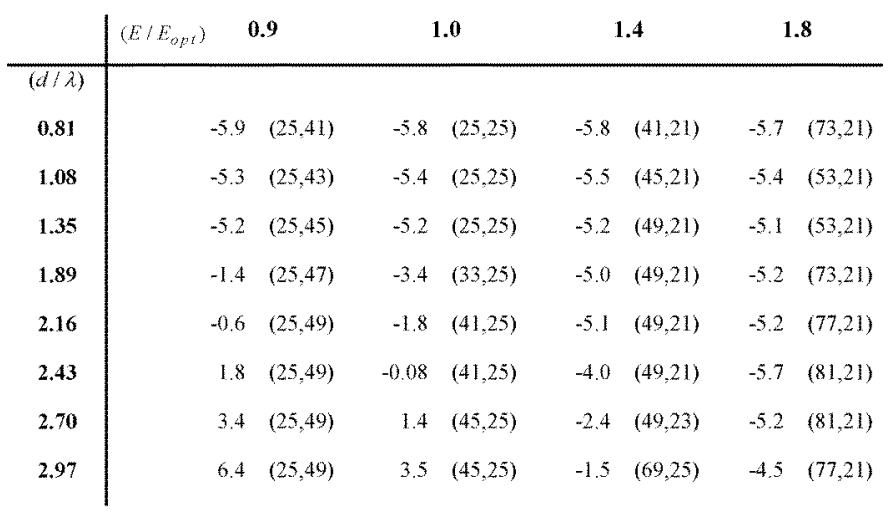

$d=D_{x}=D_{y}, \xi=\eta=\zeta=0.1 \lambda$

TABLE II

$\log _{10}$ OF RELATIVE DifFERENCE BETWEEN THE Results OBTAINED By THE EWALD MEHOd AND THE SHANKS' TRANSFORM FOR $G$ OF (4) FOR VARIOUS VALUES OF $E$

\begin{tabular}{l|llllllll} 
& $\left(E / E_{O F,}\right)$ & 0.9 & 1.0 & 1.4 & \multicolumn{1}{l}{$\mathbf{1 . 8}$} \\
\hline$(d / \lambda)$ & & & & & & & & \\
$\mathbf{0 . 8 1}$ & -4.9 & $(101,209)$ & -4.9 & $(117,117)$ & -4.9 & $(223,81)$ & -4.9 & $(437,73)$ \\
$\mathbf{1 . 0 8}$ & -4.6 & $(117,223)$ & -4.7 & $(117,117)$ & -4.7 & $(259,81)$ & -4.7 & $(379,73)$ \\
$\mathbf{1 . 3 5}$ & -4.6 & $(117,239)$ & -4.8 & $(117,117)$ & -4.8 & $(279,81)$ & -4.8 & $(393,73)$ \\
$\mathbf{1 . 8 9}$ & -2.1 & $(117,269)$ & -3.3 & $(171,125)$ & -4.3 & $(311,81)$ & -4.2 & $(553,73)$ \\
$\mathbf{2 . 1 6}$ & -0.5 & $(125,311)$ & -2.5 & $(215,125)$ & -4.8 & $(327,81)$ & -4.8 & $(581,73)$ \\
$\mathbf{2 . 4 3}$ & 1.4 & $(133,311)$ & -1.1 & $(195,125)$ & -4.8 & $(327,81)$ & -4.9 & $(633,73)$ \\
$\mathbf{2 . 7 0}$ & 4.4 & $(125,327)$ & 1.1 & $(251,125)$ & -2.3 & $(335,91)$ & -4.5 & $(657,73)$ \\
$\mathbf{2 . 9 7}$ & 5.5 & $(125,335)$ & 3.04 & $(267,125)$ & -2.1 & $(425,101)$ & -4.8 & $(653,73)$
\end{tabular}

$d=D_{x}=D_{y}=D_{z}, \xi=\eta=\zeta=0.1 \lambda$

This parameter appears incorrectly in [2] because of a misprint. In some papers dealing with the Ewald method [7]-[9], the $E_{\text {opt }}$ given in [2] is used. In [10], the value of $E$ used is not given. Since there appears to not be an expression for the optimum parameter available in the literature for 3-D arrays, we present the expression

$$
E_{o p t}=\left(\pi^{2} \frac{1 / D_{x}^{2}+1 / D_{y}^{2}+1 / D_{z}^{2}}{D_{x}^{2}+D_{y}^{2}+D_{z}^{2}}\right)^{1 / 4}
$$

which is used to balance the convergence rate between the two series in (10) and (12) for small periodic spacing. The derivation of (14) is given in [11].

\section{RESULTS}

Tables I and II present the $\log _{10}$ of the relative difference between the numerical results obtained by the application of the Shanks' transform and the Ewald method, for various values of $E$, for the computation of the series given in (1) and (4), respectively. All calculations were performed using single precision 
arithmetic in FORTRAN 77 on a DEC ALPHA processor. In the tables, the quantities in the parenthesis are the number of terms required in the summation of the spectral and the spatial series to achieve a relative change in the partial sums of less than $10^{-5}$. Also, the complex error function needed in each series was computed using the algorithms given in [12] and [13]. Inspection of data in both tables reveals that values of $E$ equal to or less than $E_{\text {opt }}$ do not work once the periodic spacing gets large. One can remedy this problem by increasing $E$ a sufficient amount, which depends on the spacing, beyond the value of $E_{\text {opt }}$. This increase in $E$ causes the number of terms needed to sum the spectral series to increase and the number of terms needed to sum the spatial series to decrease. Even though the convergence rates of the two series are unbalanced as a result of increasing $E$, the total number of terms needed in the Ewald method is still much, much smaller than that needed by the Shanks' transform. For example, in the calculation of the series (1) for a periodic spacing of $d=2.97 \lambda$ the Shanks' transform, with a convergence factor of $\varepsilon_{c}=10^{-5}$ [5], needs 19321 terms. This number is very large compared to the 98 terms needed in the Ewald method with $E / E_{o p t}=1.8$ (see Table I). One notes from the data in the tables that $E=E_{\text {opt }}$ fails to be sufficient when the periodic spacing is large enough that the two series exhibit an unequal convergence rate.

The reasons why $E$ less than or equal to $E_{\text {opt }}$ does not work for larger periodic spacing can be explained as follows. For large argument the complementary error function behaves as $\exp \left(-z^{2}\right) / z$ and this is the reason for using this method for the calculation of the FSPGF. For large periodic spacing $E_{\text {opt }}$ given by (13) and/or (14) becomes small and the imaginary part of the argument of $\operatorname{erfc}(\cdot)$ becomes large and dominant for the first several terms of the series. As a consequence, $\operatorname{erfc}(\cdot)$ becomes very large and therefore one gets very large numbers for the first several terms of these series. Similar comments can be made for the series $G_{1}$ of (10) that includes the Gaussian function directly. Gaussian decay is again achieved for terms with large indices and the two series $G_{1}$ and $G_{2}$ converge to very large nearly- equal-in-magnitude numbers of opposite sign. Since the values of the spectral and the spatial sums are very, very large but have opposite sign, one suffers severe accuracy loss upon adding the sums of the two series due to finite precision. The result is the apparent convergence to incorrect values as demonstrated in Tables I and II. By increasing $E$ beyond $E_{\text {opt }}$, one obtains small values for the imaginary part of the argument of the complementary error function and small positive values for $-\alpha_{m n p}^{2} / E^{2}$ in (10) for the first several terms of the series. As a result, one avoids the loss of accuracy resulting from adding numbers that are of nearly equal magnitude but of opposite sign and a correct sum is obtained for $G$.

By examining the data in the tables, one can see the effects of the periodic spacing on the results obtained by the use of the Ewald method. The importance of adjusting $E$ so that the method may be applied for large structure periodicity becomes apparent if one chooses to use this method for acceleration (1) of (4) in waveguide and cavity problems. By the application of the image theory to get a periodic array of cells, one may have relatively large structure periodicity and by not using a suitable value for the splitting parameter $E$, incorrect results may be obtained. Therefore, in general, an arbitrary value for the splitting parameter should not be used in the Ewald method and for some array spacing the optimal value is not suitable. One can detect situations where $E=E_{\text {opt }}$ is not suitable by looking for an imbalance in the convergence rates of the spectral and spatial series.

\section{CONCLUSIONS}

This letter has demonstrated how the periodicity of a structure affects the results obtained by computations with the Ewald method. Also, the importance of carefully selecting the value of the splitting parameter $E$ is shown. We presented an expression for optimum splitting parameter for the 3-D free-space periodic Green's function and we also illustrated the correct usage of the Ewald method for problems having large periodic spacing.

\section{REFERENCES}

[1] P. P. Ewald, "Die berechnung optischer und electrostatischer gitterpotentiale," Ann. Phys., vol. 64, pp. 253-287, 1921.

[2] K. E. Jordan, G. R. Richter, and P. Sheng, "An efficient numerical evaluation of the Green's function for the Helmholtz operator on periodic structures," J. Comp. Phys., vol. 63, pp. 222-235, 1986.

[3] T. F. Eibert, J. L. Volakis, D. R. Wilton, and D. R. Jackson, "Hybrid FE/BI modeling of 3-D doubly periodic structures utilizing triangular prismatic elements and an MPID formulation accelerated by the Ewald transformation," IEEE Trans. Antennas Propagat., vol. 45, pp. 843-850, May 1999.

[4] M. J. Park, J. Park, and S. Nam, "Efficient calculation of the Green's function for the rectangular cavity," IEEE Microwave Guided Wave Lett., vol. 8, pp. 124-126, March 1998.

[5] S. Singh, W. F. Richards, J. R. Zinecker, and D. R. Wilton, "Accelerating the convergence of series representing the free space periodic Green's function," IEEE Trans. Antennas Propagat., vol. 38, pp. 1958-1962, December 1990

[6] P. Wynn, "On a device for computing the $e_{m}\left(S_{n}\right)$ transformation," Math. Tables Aids Comput., vol. 10, pp. 91-96, 1956.

[7] M. J. Park and S. Nam, "Efficient calculation of the Green's function in rectangular waveguides," in Proc. IEEE Antennas Propagat. Soc. Int. Symp., Montreal, P.Q., Canada, July 1997, pp. 2354-2357.

[8] - "Rapid summation of the Green's for the rectangular waveguide," IEEE Trans. Microwave Theory Tech., vol. 46, pp. 2164-2166, December 1998.

[9] _ - "Rapid calculation of the Green's function in the shielded planar structures," IEEE Microwave Guided Wave Lett., vol. 7, pp. 326-328, October 1997.

[10] — - "Efficient calculation of the Green's function for multilayered planar periodic structures," IEEE Trans. Antennas Propagat., vol. 46, pp. 1582-1583, October 1998.

[11] A. Kustepeli, "Analysis and implementation of the Ewald method for waveguide and cavity structures," Ph.D. dissertation, Clemson University, Clemson, SC, December 1999.

[12] G. P. M. Poppe and C. M. J. Wijers, "More efficient computation of the complex error function," ACM Trans. Math. Softw., vol. 16, pp. 38-46, 1990.

[13] — "Algorithm 680-More efficient computation of the complex error function," ACM Trans. Math. Softw., vol. 16, p. 47, 1990. 